\title{
Innovative Approaches to Combat COVID-19 Pandemic: An Overview
}

\author{
Khursheed UI Islam and Jawed Iqbal* \\ Multidisciplinary Centre for Advanced Research and Studies, Jamia Millia Islamia, New Delhi, \\ India
}

*Corresponding author: Jawed Iqbal, PhD, Multidisciplinary Center for Advanced Research and Studies, Jamia Millia Islamia, Jamia Nagar, New Delhi-110025, India, Tel: 9911053902; Email: jiqbal1@jmi.ac.in

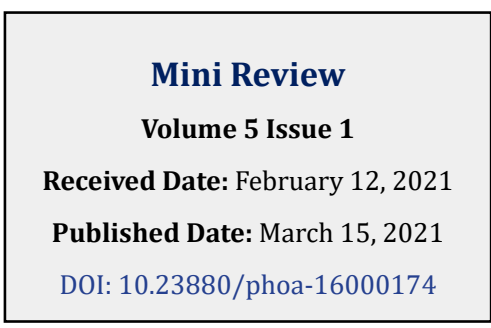

Mini Review

Volume 5 Issue 1

Published Date: March 15, 2021

DOI: $10.23880 /$ phoa-16000174

\section{Abstract}

Novel coronavirus disease-19 (COVID-19) that emerged in late 2019 in Wuhan China had caused an unprecedented crisis in health and economy. In this scenario, innovative solutions become imperative, given the less availability of resources and an immediate need for healthcare, medical supplies and support. World has witnessed a rapid response against this disease since its onset. Such kind of rapid response was possible due to certain innovative approaches which assisted in timely tracing, diagnosing and treatment of COVID-19 patients. Utilization of innovative technologies for population screening, simple and advanced technologies for rapid and reliable diagnosis and nanotechnology for vaccine development are some of the important highlights to be mentioned. Collaborations within scientific community and production of an immense amount of knowledge have hardly been known to world before COVID-19 pandemic. This mini-review is mainly focused on scientific innovations and technologies that aided in responding against COVID-19 and will also highlight the importance of scientific collaborations and data availability during the COVID-19 pandemic.

Keywords: SARS-CoV-2; COVID-19; Diagnostics Tools; Vaccine; Repurposing Drugs

Abbreviations: COVID-19: Coronavirus disease 19; SARSCoV-2: Severe Acute Respiratory Syndrome Coronavirus 2; ECDC: European Center for Disease Prevention and Control; CRISPR: Clustered Regularly Interspaced Short Palindromic Repeats; RT-LAMP: Reverse Transcriptase Loop mediated Isothermal Amplification; SHERLOCK: Specific High-sensitivity Enzymatic Reporter un-Locking; CARMENCas13a: Combinatorial Arrayed Reactions for Multiplexed Evaluation of Nucleic Acids; VIRRION: Virus captured with rapid Raman spectroscopy detection and identification; RTPCR: Reverse Transcriptase Polymerase Chain Reaction; CASSPIT: Cas13 Assisted Saliva based and Smart Phone Integrated Testing; CSIR-IGIB: Council for Scientific and Industrial Research- Institute of Genomics and Integrative Biology.

\section{Novel Approaches for Community Screening and Prediction of Viral Transmission Rates}

The biggest challenge during the onset of COVID-19 was the contact tracing and screening of population for Severe Acute Respiratory Syndrome Coronavirus 2 (SARSCoV-2) infection. Spreading of SARS-CoV-2 via asymptomatic carries made contact tracing a challenging task for the health workers, thus urged scientists to look for some innovative approaches to assist in stringent tracing and monitoring of COVID-19 positive patients. Many Smartphone applications were developed in different countries for contact tracing, tracking of individuals, training of healthcare workers which include Aarogya situ, Apple COVID-19, COVID-UAE, Apollo COVID-19, ECDC threat reports and Pakistan's National Action 
Plan for COVID-19 etc [1]. Contact tracing helped in immediate isolation of positive patients from normal population. Moreover, machine learning, artificial intelligence and deep learning tools have enabled the study of large datasets that have helped to understand fundamental knowledge regarding this virus [2], same approach can be used in future to strengthen the basic research.

\section{Revolutions in COVID-19 Diagnosis}

Easy and affordable diagnosis is an important parameter in dealing with a novel pathogen like SARS-CoV-2. Since RTPCR has been well accepted as a gold standard for pathogen diagnosis but COVID-19 pandemic urged the use of different tools which are easily accessible and affordable technologies to help in rapid diagnosis of COVID-19. CRISPR technology, nanotechnology, RT-LAMP etc were exploited to develop diagnostic kits for rapid diagnosis of SARS-CoV-2. SHERLOCK Bioscience was the first approval of CRISPR-based diagnostic by FDA which provides test results in one hour. Other CRISPRbased diagnostic methods that are under development include CARMEN-Cas13a (Combinatorial Arrayed Reactions for multiplexed Evaluation of Nucleic Acids) by Broad Institute and Harvard University, and CRISPR-Cas13based electrochemical microfluidic sensor by University of Freiburg, Germany VIRRION (Virus captured with rapid Raman spectroscopy detection and identification) [3]. Researchers also employed CRISPR-Cas13a for low cost and rapid screening of SARS-CoV-2 from the nasal swabs of the patients where the results can be visualized by mobile phone microscopy [4]. Certain CRISPR-based diagnostic technology that is believed to be parallel in sensitivity with RT-PCR based diagnosis is CASSPIT, a Cas13a-based diagnostic kit developed by MCARS, Jamia Millia Islamia, India [5]. Furthermore, FnCas9 Editor Linked Uniform Detection Assay (FELLUDA) has been developed by CSIR-IGIB Delhi, India [6]. Many other potential diagnostic approaches that had been brought on table by various scientists, as reviewed by Islam, et al. [7], which might add the advantage of rapid, affordable and accurate diagnosis in future. Another novel approach employing nanoparticles for stringent detection of pathogen like SARS-CoV-2 was demonstrated recently [8]. There are many more such new approaches that have potential to become rapid diagnostic approaches in pandemic or epidemic health crisis.

\section{Healthcare Innovations Inspired by Covid-19 Pandemic}

Ideally it takes years to develop methodologies and technologies in healthcare system and also millions and billions are spent to make these technologies ready for public use. However, a global pandemic like COVID-19 that has swept through the population and has inspired innovative healthcare approaches which has changed this typical timeline of years into weeks. Moreover, T plastic cube designed to cover head of the patient with two arm holes for performing intubations during handling the COVID-19 patient [9]. Building Fangcang hospitals in China was a novel approach to respond to COVID-19 pandemic, these were implemented first time in China in February 2020 to tackle the SARS-CoV-2 outbreak [10]. India and Pakistan responded to COVID-19 crisis by making trains as the hospital wards that was an extraordinary move to combat SARS-CoV-2 spread [3]. Moreover, drug repurposing was another approach to combat SARSCoV-2 infection, which allows an emergency use of an existing (non-specific) drug, in this approach an existing drug (not specific for the pathogen) is tried for the novel pathogen based on its computational screening and docking results. Combination of Hydroxylchloroquine and Azithromycin was used as a repurposed drug against COVID-19 prophylaxis [11]. Another such approach, where a combination of existing antivirals is being proposed to work against SARS-CoV-2 as suggested by research conducted by Bhat, et al. [12] these approaches can help in basic understanding of novel therapeutics against pathogenic diseases.

\section{Rapid Development of COVID-19 Vaccines}

COVID-19 pandemic has witnessed a rapid vaccine development since its inception. Just after the availability of sequence data of SARS-CoV-2, research on vaccines was eventually started in different countries which led to the early development of various vaccines against this pathogen [13]. World has never experienced such a rapid development of vaccines against any other pathogenic diseases. Usually it takes around 20 years to develop any sustainable vaccine against a pathogen but severity of SARS-CoV-2 spread in the population prompted the early development of COVID-19 vaccines [14]. According to the WHO updates around 63 vaccines are in clinical trials (early or advanced) and 177 vaccine candidates are in preclinical development in academia and industry (https://www.who. int/publications/m/item/draft-landscape-of-covid-19candidate-vaccines). There are different generic platforms which have been employed to develop vaccines against COVID-19 including nucleic acid vaccines (RNA/DNA), inactivated viral vaccines, live attenuated vaccines, vectorbased vaccines, protein subunit vaccines etc [15]. Among the frontrunners, it is important to mention that there are mRNA based vaccines such as BNT162b2 (Pfizer/Biontech) [16] and mRNA-1273 (Moderna), [17] as well as others like Adenoviral vaccine, Covishield (Oxford University) [18] and inactivated virus vaccines like Coronavac (Sinovac Biotech China) [19], and Covaxin (Bharat Biotech/ICMR) [20]. 


\section{Conclusion}

The global challenges experienced by world during the COVID-19 pandemic have inspired creative and innovative approaches to deal with its severity and consequences. Technological innovations had somehow helped frontline workers to deal with COVID-19 patients. Innovation in diagnostic approaches like SHERLOCK, CRISPR technologies have taken the diagnostics to a next level by providing easy, rapid, affordable and reliable diagnostic approaches to combatSARS-CoV-2 spread. The pace of research on COVID-19 and research collaborations has signified the strength of research round the globe. Public-private partnership has been the hallmark for speeding up the promising treatments and COVID-19 vaccine development. The global pandemic further highlights the importance of research and funding for disaster management and public health emergency.

\section{Acknowledgment}

The support of Ramalingaswami Fellowship grant (BT/ RLF/Re-entry/09/2015) from Department of Biotechnology (DBT) and the support of Early Career Research Award grant (File No. ECR/2018/002114) from Science and Engineering Research Board (SERB), Department of Science \& Technology, Government of India to author JI is acknowledged.

\section{References}

1. Davalbhakta S, Shailesh A, Shobhit K, Vishwesh A, Samruddhi B, et al. (2020) A Systematic Review of Smartphone Applications Available for Corona Virus Disease 2019 (COVID19) and the Assessment of Their Quality Using the Mobile Application Rating Scale (MARS). J Med Syst 44(9): 164.

2. Tsikala VM, Eleftheria A, Joanna G, Fadi S, Evangelia KM, et al. (2020) Emerging Technologies for Use in the Study, Diagnosis, and Treatment of Patients with COVID-19. Cell Mol Bioeng 13(4): 1-9.

3. Harris M, Yasser B, Jim B, Dhananjaya S (2020) Fast and Frugal Innovations in Response to the COVID-19 Pandemic. Nat Med 26(6): 814-817.

4. Fozouni P, Sungmin S, Maria DLD, Gavin JK, Carley NG, et al. 2021. Amplification-Free Detection of SARS CoV-2 with CRISPR-Cas13a and Mobile Phone Microscopy. Cell 184(2): 323-333.

5. Azmi I, Faizan MI, Rohit K, Siddharth RY, Nisha C, et al. (2020) A Saliva-Based RNA Extraction-Free Workflow Integrated with Cas13a for SARS-CoV-2 Detection. MedRxiv, pp: 1-39.
6. Azhar M, Rhythm P, Manoj K, Asgar HA, Riya R, et al. (2020) Rapid, Accurate, Nucleobase Detection Using FnCas9. Medrxiv, pp: 1-28.

7. Islam KUI, Jawed (2020) An Update on Molecular Diagnostics for COVID 19. Front Cell Infect Microbiol 10: 560616.

8. Zhu X, Xiaoxia W, Limei H, Ting C, Licheng W, et al. (2020) Reverse Transcription Loop-Mediated Isothermal Amplification Combined with Nanoparticles-Based Biosensor for Diagnosis of COVID-19. medRxiv, pp: 1-19.

9. Everington K (2020) Taiwanese Doctor Invents Device to Protect US Doctors against Coronavirus.

10. Chen S, Zongjiu Z, Juntao Y, Jian W, Xiaohui Z (2020) Fangcang Shelter Hospitals: A Novel Concept for Responding to Public Health Emergencies. The Lancet 395(10232): 1305-1314.

11. Gautret P, Jean CL, Philippe P, Van TH, Line M, et al. (2020) Hydroxychloroquine and Azithromycin as a Treatment of COVID-19: Results of an Open-Label Non-Randomized Clinical Trial. Int J Antimicrob Agents 56(1): 105949.

12. Bhat ZA, Dheeraj C, Jawed I, Arumugam M, Sanjeev BS (2020) Targeting Allosteric Pockets of SARS-CoV-2 Main Protease Mpro. ChemRxiv, pp: 1-81.

13. Lurie N, Melanie S, Richard H, Jane H (2020) Developing Covid-19 Vaccines at Pandemic Speed. N Engl J Med 382(21): 1969-1973.

14. Gouglas D, Tung TLe, Klara H, Aristidis K, Trygve D, et al. (2018) Estimating the Cost of Vaccine Development against Epidemic Infectious Diseases: A Cost Minimisation Study. The Lancet Global Health 6(12): e1386-1396.

15. Locht C (2020) Vaccines against COVID-19. Anaesth Crit Care Pain Med 39(6): 703-705.

16. Polack FP, Stephen JT, Nicholas Kitchin, Judith A, Alejandra G, et al. (2020) Safety and Efficacy of the BNT162b2 mRNA Covid-19 Vaccine. N Engl J Med 383(27): 26032615.

17. Corbett KS, Barbara F, Kathryn EF, Joseph RF, Seyhan BB, et al. (2020) Evaluation of the mRNA-1273 Vaccine against SARS-CoV-2 in Nonhuman Primates. New England Journal of Medicine 383: 1544-1555. 
18. Jia W, Rudragouda C, Chao Z, Mingxi Li, Haixia Z, et al. (2019) Single Intranasal Immunization with Chimpanzee Adenovirus-Based Vaccine Induces Sustained and Protective Immunity against MERS-CoV Infection. Emerg Microbes Infect 8(1): 760-772.

19. Zhang W, Rong HD, Bei Li, Xiao SZ, Xing LY, et al. (2020) Molecular and Serological Investigation of 2019-NCoV Infected Patients: Implication of Multiple Shedding
Routes. Emerg Microbes Infect 9(1): 386-389.

20. Philbin VJ, David JD, Leighanne CG, Guadalupe C, Zhen Tan, et al. (2012) Imidazoquinoline Toll-like Receptor 8 Agonists Activate Human Newborn Monocytes and Dendritic Cells through Adenosine-Refractory and Caspase-1-Dependent Pathways. J Allergy Clin Immunol 130(1): 195-204. 